\title{
Recent results on exclusive production at HERA
}

\author{
Mariusz Przybycien* (on behalf of the $\mathrm{H} 1$ and ZEUS Collaborations) \\ AGH University of Science and Technology \\ Faculty of Physics and Applied Computer Science \\ Al. Mickiewicza 30, 30-059 Cracow, Poland \\ E-mail: mariusz.przybycien@agh.edu.pl
}

Exclusive processes at HERA can lead to a deeper insight into production mechanisms in QCD. A series of processes is presented here. A first measurement is presented of exclusive photoproduction of $\rho^{0}$ mesons associated with leading neutrons at HERA. The H1 data are interpreted in terms of a double peripheral process, involving pion exchange at the proton vertex followed by elastic photoproduction of a $\rho^{0}$ meson on the virtual pion. The value of the elastic cross section indicates significant absorptive corrections for the exclusive reaction. Production of exclusive dijets in diffractive deep inelastic $e^{ \pm} p$ scattering has been measured by ZEUS. The results are compared to predictions from models based on different assumptions about the nature of the diffractive exchange. The exclusive deep inelastic electroproduction and photoproduction of $\psi(2 S)$ and $J / \psi(1 S)$ has been studied by ZEUS. The cross-section ratio $\sigma(\psi(2 S)) / \sigma(J / \psi(1 S))$ has been measured as a function of $Q^{2}, W$ and $t$. The results are compared to many predictions of QCD-inspired models of exclusive vector-meson production.

38th International Conference on High Energy Physics

3-10 August 2016

Chicago, USA

${ }^{*}$ Speaker. 


\section{Introduction}

Measurements of exclusive processes are presented below based on data collected by the H1 and ZEUS experiments at the HERA Collider, where beams of electrons/positrons of energy 27.5 $\mathrm{GeV}$ were collided with protons of energy $920 \mathrm{GeV}$. This corresponds to the energy available in the centre-of-mass system equal to $\sqrt{s}=318 \mathrm{GeV}$.

\section{Exclusive production at HERA}

Exclusive photoproduction of the $\rho^{0}$ meson with a leading neutron has been studied by the H1 Collaboration [1]. In the Regge framework this process is interpreted as a Double-Regge-pole exchange reaction and is schematically shown in Fig. 1(left). The process can also be seen as a proton dissociating into $\left(n, \pi^{+}\right)$system which then scatters elastically on the $\rho^{0}$ via exchange of the Regge trajectory with the vacuum quantum numbers, called the Pomeron. The analysis is based on an $e p$ data sample corresponding to an integrated luminosity of $1.16 \mathrm{pb}^{-1}$. The photon virtuality in this analysis is limited to $Q^{2}<2 \mathrm{GeV}^{2}$ with an average value of $0.04 \mathrm{GeV}^{2}$. In the following account, the kinematical variables $t, t^{\prime}, W_{\gamma p}, W_{\gamma \pi}$ and $x_{L}$ shown in Fig. 1(left) have their usual meaning. Most of the experimental properties of this reaction are successfully explained by the generalised DrellHiida-Deck model [2,3], in which in addition to the pion exchange two further contributions are included: (a) neutron exchange, where the roles of the neutron and $\pi^{+}$in Fig. 1(left) are replaced, and (b) the direct pole, where the proton interacts directly with $\rho^{0}$ via Pomeron exchange and the excited proton subsequently dissociates in $\left(\pi^{+}, n\right)$ system. These two processes contribute to the total scattering amplitude with similar magnitude but opposite sign. Therefore they largely cancel in most of the phase space, in particular at small momentum transfer squared at the proton vertex, $t \rightarrow 0$, such that the pion exchange diagram dominates the cross section.

The $\rho^{0}$ candidate selection requires the reconstruction of the trajectories of two, and only two, oppositely charged particles in the central tracking detector $\left(\pi^{+}\right.$from the proton vertex is produced at a very small angle and is lost in the beam pipe). The two tracks must originate from a common vertex lying within $\pm 30 \mathrm{~cm}$ in $z$ of the nominal ep interaction point, and must have transverse momenta above $0.2 \mathrm{GeV}$ and polar angles within the interval $20^{\circ}<\theta<160^{\circ}$. Events containing a leading neutron are selected by requiring a hadronic cluster in the Forward Neutron Calorimeter
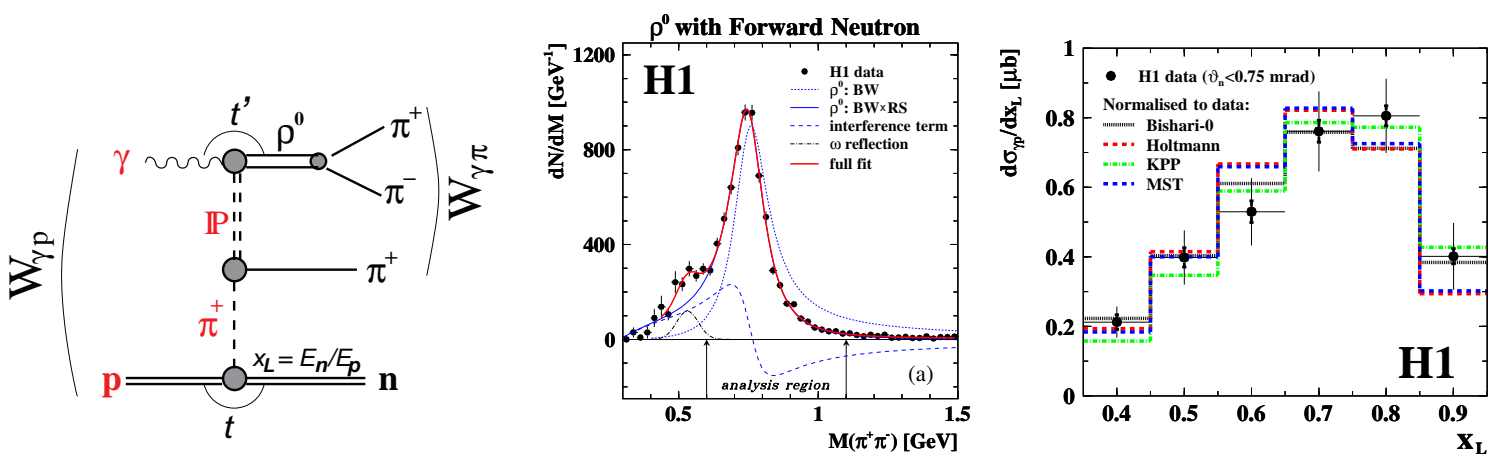

Figure 1: (left) Schematic diagram for the exclusive photoproduction of $\rho^{0}$ mesons associated with leading neutrons. (middle) Mass distribution of the $\pi^{+} \pi^{-}$system together with the fit discussed in the text. (right) Differential cross section $d \sigma_{\gamma p} / d x_{L}$ compared to the predictions based on different models of the pion flux. 

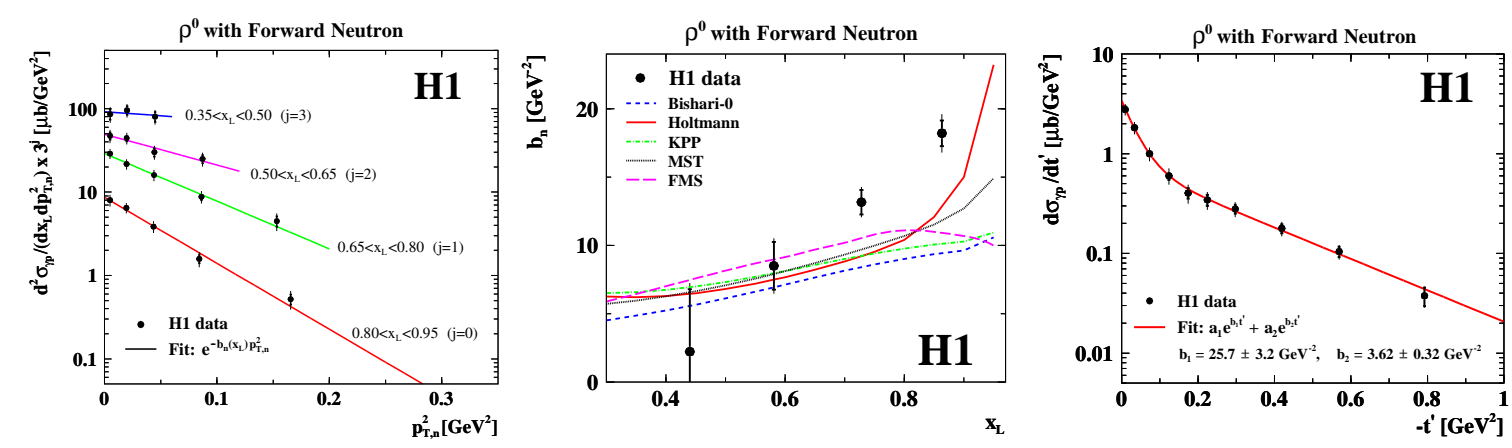

Figure 2: (left) Double differential cross section $d^{2} \sigma_{\gamma p} / d x_{L} d p_{T, n}^{2}$ fitted with exponential functions. (middle) The exponential slopes fitted through the $p_{T}^{2}$ dependence of the leading neutron as a function of $x_{L}$. (right) Differential cross section $d \sigma_{\gamma p} / d t^{\prime}$ fitted with the sum of two exponential functions.

(FNC) with energy above $120 \mathrm{GeV}$ and the polar angle below $0.75 \mathrm{mrad}$, which effectively restricts the neutron transverse momenta to the range $p_{T, n}<x_{L} \cdot 0.69 \mathrm{GeV}$. The invariant mass distribution of the two tracks under the charged pion mass hypothesis is shown in Fig. 1(middle). A fit is performed in the range $M_{\pi \pi}>0.4 \mathrm{GeV}$ using the Ross-Stodolsky parametrisation [4] for the $\rho^{0}$ meson mass shape and adding contributions for the reflection from $\omega \rightarrow \pi^{+} \pi^{-} \pi^{0}$ and for the non-resonant background. The fitted values of the resonance mass and width are in agreement with PDG values. The cross section is then calculated for the full mass range using the resonant part only, represented by the relativistic Breit-Wigner function. The Breit-Wigner shape is strongly distorted due to interference with the non-resonant $\pi \pi$ production amplitude.

In Fig. 1(right) the single differential $\gamma p$ cross section is shown as a function of $x_{L}$ and compared to predictions based on several models of the pion flux which describe the data very well. The measured double differential cross section $d^{2} \sigma_{\gamma p} / d x_{L} d p_{T, n}^{2}$ is shown in Fig. 2(left). The $p_{T, n^{-}}^{2}$ distributions measured for fixed $x_{L}$ are compatible with an exponential shape within the statistical and uncorrelated systematic errors. The cross sections are fitted by a single exponential function $e^{-b_{n}\left(x_{L}\right) p_{T, n}^{2}}$ in each $x_{L}$ bin. The results of the fits are presented in Fig. 2(middle) and compared to the $b$-slopes obtained from several pion flux parametrisations. Despite of the large experimental uncertainties none of the models is able to reproduce the data. The $\gamma p$ cross section as a function of four-momentum transfer squared of the $\rho^{0}$ meson, $t^{\prime}$, is shown in Fig. 2(right). One can see the very pronounced feature of a strongly changing slope between low- $t^{\prime}$ and high- $t^{\prime}$ regions. The fit was performed to the sum of two exponents as shown in the figure. In a geometric picture the large value of $b_{1}$ suggests that for a significant part of the data $\rho^{0}$ mesons are produced at large impact parameter values of order $\left\langle r^{2}\right\rangle=2 b_{1}(\hbar c)^{2} \simeq 2 \mathrm{fm}^{2} \approx\left(1.6 R_{p}\right)^{2}$. Effectively this means photons find pions in a cloud which extends far beyond the proton radius $R_{p}$.

First measurement of exclusive dijet production in diffractive deep inelastic ep collisions has been performed by the ZEUS experiment [5]. The process is schematically shown in Fig. 3(left). Symbols in parentheses denote four-momenta of the particles, and the kinematical variables: $Q^{2}$, $s, W, t$ shown in the figure have their usual meaning. The process can be viewed as an interaction of virtual photon and the proton via exchange of a colourless object known as Pomeron. The same process in the $\gamma^{\star}-I P$ centre-of-mass frame is shown in Fig. 3(middle), where definitions of lepton and jet planes are introduced. The production of exclusive dijets in DIS is sensitive to the nature of the object exchanged between the virtual photon and the proton. Single differential cross sections 

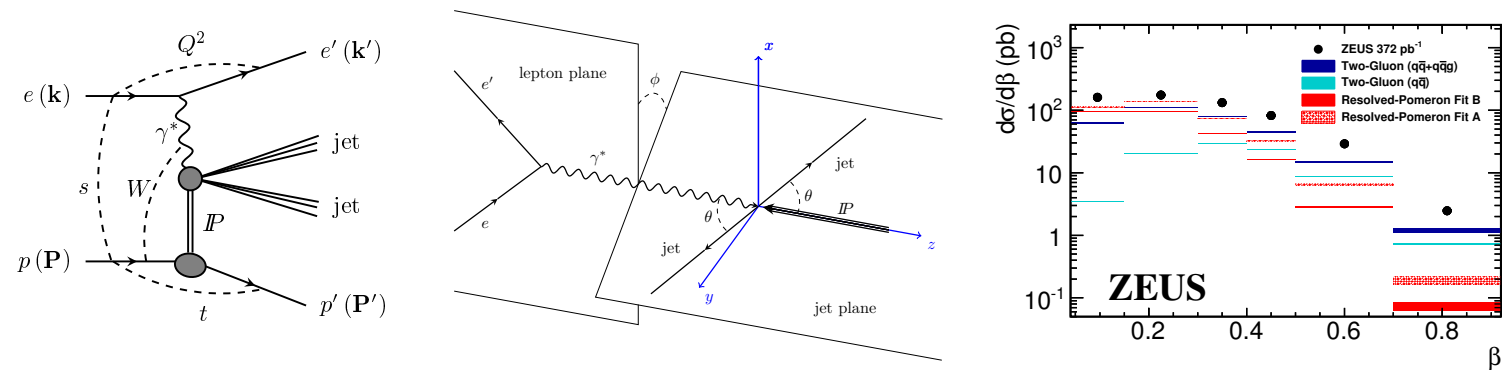

Figure 3: (left) Schematic view of the diffractive production of exclusive dijets in $e p$ DIS. (middle) Definitions of lepton and jet planes and the angles $\phi$ and $\theta$ in $\gamma^{\star}-I P$ CMS frame. (right) Differential cross section for dijet production as a function of $\beta$ compared to model predictions.

for dijet production as a function of $\phi$ in $k_{t}$-factorisation [6] and collinear factorisation [7] are proportional to $1+A\left(p_{\mathrm{T}, j e t}\right) \cos 2 \phi$. the parameter $A$ is expected to be positive if the quark-antiquark pair is produced via the interaction of a single gluon with the virtual photon and negative if the system of two gluons takes part in the interaction.

The analysis is based on data collected by the ZEUS experiment corresponding to an integrated luminosity of $372 \mathrm{pb}^{-1}$. The DIS events were required to have a scattered electron with energy $E_{e}^{\prime}>10 \mathrm{GeV}$ observed in the calorimeter with additional constraints set on $Q^{2}>25 \mathrm{GeV}^{2}$ and $90<W<250 \mathrm{GeV}$. Diffractive events were identified with help of the large rapidity gap method with pseudorapidity of a most forward calorimeter object $\eta_{\max }<2$ with additional restriction on the invariant mass of the diffractive system $M_{X}>5 \mathrm{GeV}$ and $x_{I P}<0.01$. The $k_{T}$-cluster algorithm in exclusive mode (all final state objects are clustered) was used to reconstruct two jets with $p_{\mathrm{T}, \text { jet }}>2 \mathrm{GeV}$. The measured cross sections have been corrected for the contribution from proton dissociation estimated to be at the level of $45 \%$. The cross section as a function of $\beta$, the fraction of the longitudinal momentum of the Pomeron taken by the struck quark, is compared in Fig. 3(right) to the model predictions. The Resolved-Pomeron model [8] predicts faster decrease of the cross section with $\beta$ than observed in data. The Two-Gluon-Exchange model prediction describes the shape of the measured $\beta$ distribution reasonably well, however underestimates the total normalisation by factor almost two.

The cross section for dijet production has been also measured as a function of $\phi$ in several intervals of $\beta$. In Fig. 4 (left and middle) two examples are shown together with models predictions and a theory motivated fit of the function $1+A\left(p_{\mathrm{T}, j e t}\right) \cos 2 \phi$. The values of the parameter $A$ obtained from the fits are shown as a function of $\beta$ in Fig. 4(right). The Two-Gluon-Exchange model predicts reasonably well the measured values of $A$, whereas the Resolved-Pomeron model exhibits a different trend.

A measurement of the ratio of the cross sections for the reactions $\gamma^{\star} p \rightarrow \psi(2 S)+Y$ and $\gamma^{\star} p \rightarrow J / \psi(1 S)+Y$, where $Y$ denotes either a proton or a low mass proton dissociative system has been performed by the ZEUS experiment [9]. The $\psi(2 S)$ and the $J / \psi(1 S)$ mesons have the same quark content, but different radial distributions of the wave functions, and their mass difference is small compared to the HERA centre-of-mass energy. Therefore this measurement allows QCD predictions of the wave function dependence of the $c \bar{c}$-proton cross section to be tested.

A suppression of the $\psi(2 S)$ cross section relative to the $J / \psi(1 S)$ is expected, as the $\psi(2 S)$ wave function has a radial node close to the typical transverse separation of the virtual $c \bar{c}$ pair. 

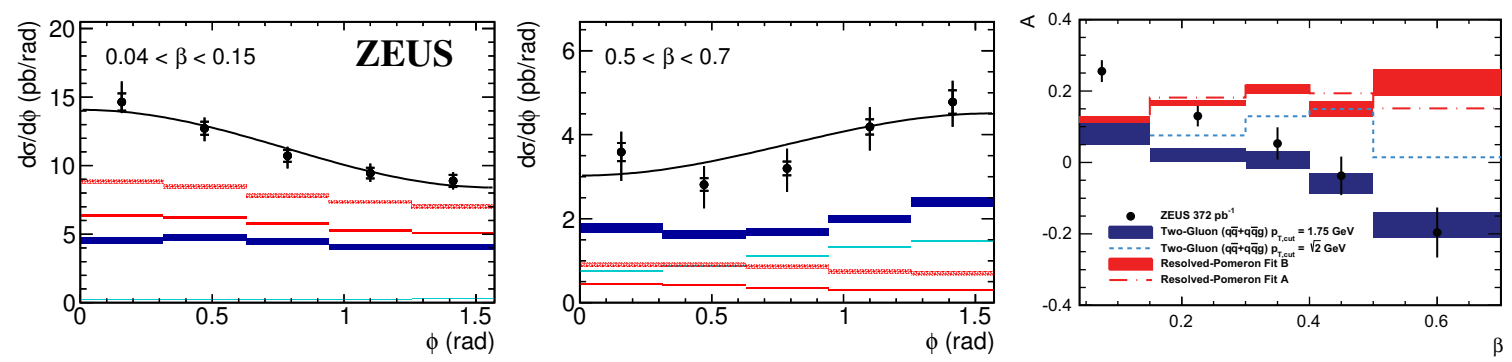

Figure 4: (left, middle) Differential cross section for dijet production as a function of $\phi$ is shown in two $\beta$ intervals together with the results of a fit of the function $1+A\left(p_{\mathrm{T}, j e t}\right) \cos 2 \phi$. (right) Values of the parameter $A$ obtained from the fits are shown as a function of $\beta$. Data are compared to model predictions.

The ratio of the cross sections $\sigma(e p \rightarrow e \psi(2 S) Y) / \sigma(e p \rightarrow e J / \psi(1 S) Y)$ has been measured in the kinematic range of $5<Q^{2}<80 \mathrm{GeV}^{2}, 30<W<210 \mathrm{GeV}$ and $|t|<1 \mathrm{GeV}^{2}$ using data corresponding to the luminosity of $468 \mathrm{pb}^{-1}$. An additional sample of $114 \mathrm{pb}^{-1}$ was used to extend the measurement to the range $2<Q^{2}<5 \mathrm{GeV}^{2}$. Events were required to have no activity in the central ZEUS detector in addition to signals from the scattered electron and the decay products of the $\psi(2 S)$ or $J / \psi(1 S)$. The decay channels used were $J / \psi(1 S) \rightarrow \mu^{+} \mu^{-}, \psi(2 S) \rightarrow \mu^{+} \mu^{-}$and $\psi(2 S) \rightarrow J / \psi(1 S) \pi^{+} \pi^{-}$with the subsequent decay $J / \psi(1 S) \rightarrow \mu^{+} \mu^{-}$.

The $\mu^{+} \mu^{-}$mass distribution for the selected events in dimuon decay channels is shown in Fig. 5(left). Clear $J / \psi(1 S)$ and $\psi(2 s)$ peaks and no other significant peaks are observed. The background was fit by straight line in the side-bands of the signals. Selection of $\psi(2 S)$ in the decay channel to $\pi^{+} \pi^{-} \mu^{+} \mu^{-}$is based on the scatter plot of $\Delta M=M_{\mu \mu \pi \pi}-M_{\mu \mu}$ versus $M_{\mu \mu}$ shown in Fig. 5(right) together with distributions of the $\Delta M$ and $M_{\mu \mu \pi \pi}$ for $3.02<M_{\mu \mu}<3.17 \mathrm{GeV}$. The $\Delta M$ distribution show a narrow peak at the nominal $\psi(2 S)-J / \psi(1 S)$ mass difference. Simultaneous cuts on $\Delta M$ and $M_{\mu \mu}$ as shown by the dashed lines in the scatter plot were used to select the $\psi(2 S) \rightarrow \mu \mu \pi \pi$ events.

The combined cross section ratio was obtained using the with the statistical uncertainties weighted average of the cross sections determined for the two $\psi(2 S)$ decay channels. The mea-

ZEUS

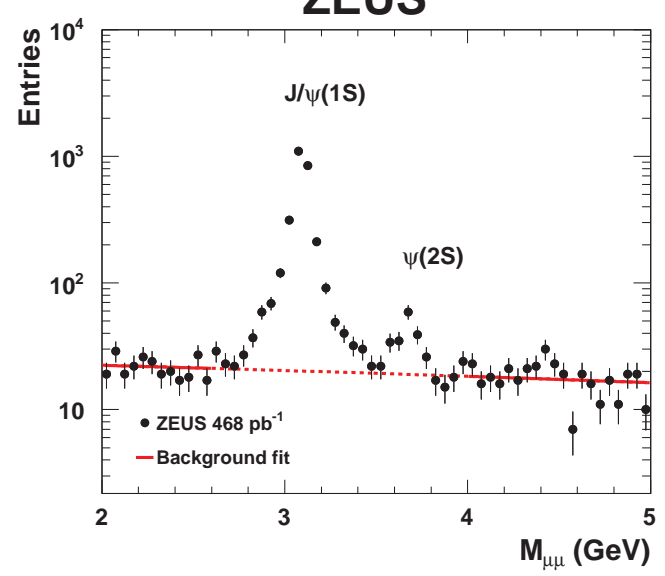

ZEUS
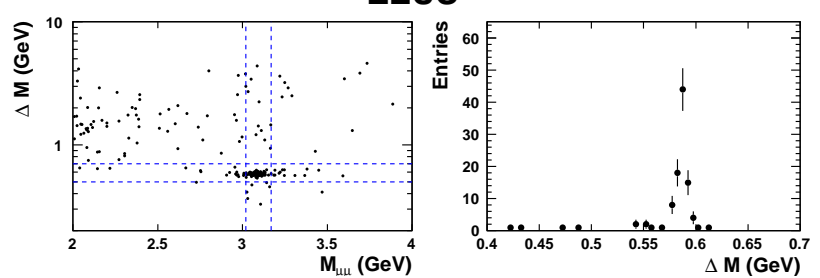

- ZEUS $468 \mathrm{pb}^{-1}$

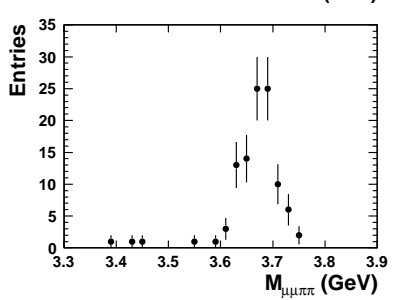

Figure 5: (left) Two-muon invariant mass distribution for exclusive dimuon events. (right) Selection of events with exclusive production of $\psi(2 S)$ meson decaying into $\pi \pi \mu \mu$ system. 

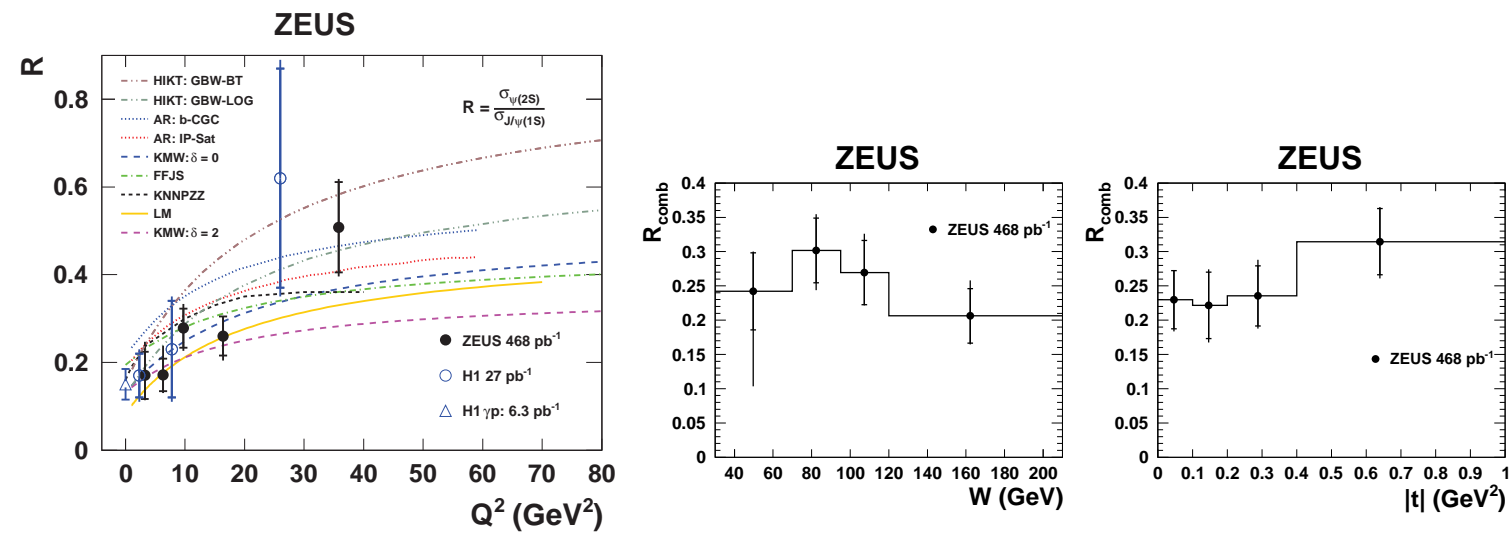

Figure 6: Cross section ratio $\sigma_{\psi(2 S)} / \sigma_{J / \psi(1 S)}$ for the combined $\psi(2 S)$ decay modes as a function of $Q^{2}$ compared to previous $\mathrm{H} 1$ results and several model predictions (left), $W$ (middle) and $|t|$ (right). The references to the $\mathrm{H} 1 \mathrm{measurements}$ and theory predictions one can find in [9].

sured ratios are shown in Fig. 6 as a function of $Q^{2}, W$ and $|t|$. The data contain a background of charmonium production with diffractive masses $M_{Y} \lesssim 4 \mathrm{GeV}$. Assuming that the cross section ratio does no vary with $M_{Y}$, the results are not affected by the proton dissociation background. The ratios as a function of $W$ and $|t|$ are compatible with a constant. For the $Q^{2}$ dependence, a positive slope is observed. The data are in good agreement with the H1 measurements as well as with most of theory predictions shown in the figure.

\section{Summary}

Recent results from the $\mathrm{H} 1$ and ZEUS experiments related to the study of exclusive processes have been briefly presented. For more details and in depth discussion of the measurements the reader is referred to the original publications.

\section{References}

[1] H1 Collaboration, V. Andreev et al., Exclusive $\rho^{0}$ meson photoproduction with a leading neutron at HERA, Eur. Phys. J. C (2016) 76:41, [hep-ex/1607.02774].

[2] S.D. Drell, K. Hiida, Phys. Rev. Lett. 7, 199 (1961).

[3] R.T. Deck, Phys. Rev. Lett. 13, 169 (1964).

[4] M. Ross, L. Stodolsky, Phys. Rev. 149, 1172 (1966).

[5] ZEUS Collaboration, H. Abramowicz et al., Production of exclusive dijets in diffractive deep inelastic scattering at HERA, Eur. Phys. J. C (2016) 76:16, [hep-ex/1505.05783].

[6] J. Bartels, H. Lotter, M. Wüsthoff, Phys. Lett. B 386, 389 (1996), [hep-ph/9605356]; J. Bartels, H. Lotter, M. Wüsthoff, Phys. Lett. B 379, 239 (1996), [hep-ph/9602363].

[7] V.M. Braun, D.Yu. Ivanov, Phys. Rev. D 72, 034016 (2005), [hep-ph/0505263].

[8] G. Ingelman, P.E. Schlein, Phys. Lett. B 152, 256 (1985)

[9] ZEUS Collaboration, H. Abramowicz et al., Measurement of the cross section ratio $\sigma_{\psi(2 S))} / \sigma_{J / \psi(1 S)}$ in deep inelastic ep scattering at HERA, Nucl. Phys. B909 (2016) 934-953, [hep-ex/1606.08652]. 